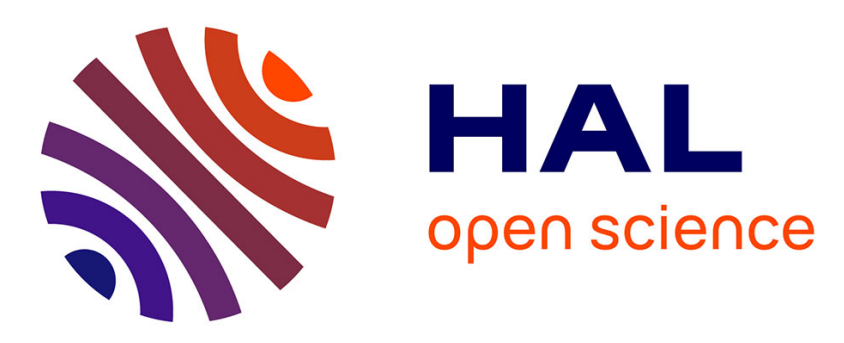

\title{
Mechanical behaviour of granular-gas and heterogeneous-fluid systems submitted to vibrations in microgravity
}

\author{
Pierre Evesque, Daniel Beysens, Yves Garrabos
}

\section{- To cite this version:}

Pierre Evesque, Daniel Beysens, Yves Garrabos. Mechanical behaviour of granular-gas and heterogeneous-fluid systems submitted to vibrations in microgravity. Journal de Physique IV Proceedings, 2001, 11 (PR6), pp.Pr6-49-Pr6-56. 10.1051/jp4:2001606 . hal-03266177

\section{HAL Id: hal-03266177 \\ https://hal.science/hal-03266177}

Submitted on 21 Jun 2021

HAL is a multi-disciplinary open access archive for the deposit and dissemination of scientific research documents, whether they are published or not. The documents may come from teaching and research institutions in France or abroad, or from public or private research centers.
L'archive ouverte pluridisciplinaire HAL, est destinée au dépôt et à la diffusion de documents scientifiques de niveau recherche, publiés ou non, émanant des établissements d'enseignement et de recherche français ou étrangers, des laboratoires publics ou privés. 


\title{
Mechanical behaviour of granular-gas and heterogeneous-fluid systems submitted to vibrations in microgravity
}

\author{
P. Evesque, D. Beysens ${ }^{1}$ and Y. Garrabos ${ }^{1}$ \\ Laboratoire MSSMat, UMR 8579 du CNRS, Ecole Centrale de Paris, 92295 Châtenay-Malabry, \\ France \\ ${ }^{1}$ ESEME-CEACNRS, ICMCB, 87 avenue du Dr. A. Schweitzer, 33608 Pessac cedex, France
}

\begin{abstract}
Recent vibration experiments (amplitude A, frequency $f=\omega / 2 \pi$ ) in microgravity have demonstrated that the "granular-gas" state exists only in the Knudsen regime. The excitation was "supersonic" and the probability density function of the pressure of the gas scaled as $(A \omega)^{3 / 2}$. This paper draw some analogy with i) the ideal-gas theory and (ii) the thermodynamics of a single particle in a $1 \mathrm{~d}$ box. The anomalous scaling of pressure fluctuations $(\mathrm{A} \omega)^{3 / 2}$ is explained by the crossover from a single-collision regime during the sampling time, which imposes $\mathrm{p}-$ $(A \omega)$ at small speed, to a multiple-collision regime imposing $p \sim(A \omega)^{2}$. The effects of air and g-jitter are discussed and quantified. Grain-grain collisions are introduced and their effect on the speed distribution discussed. The second part of the paper addresses the general problem of pattern formation under vibration and in weightlessness condition, using the example of sedimentation.
\end{abstract}

\section{INTRODUCTION}

It is a major scientific and technical goal to be able to control and handle heterogeneous fluids in weightlessness conditions. Within a few examples, this paper shows that vibration can be very helpful.

In the first part, the general problem of the behaviour of vibrated granular-gas [1-10] is addressed and the results of a micro-gravity experiment are recalled [6,7]: it is demonstrated that clustering process occurs as soon as the mean free path of the particles is smaller than the sample size. This is some kind of (gas-cluster) phase transition. It is also proved that the gas excitation is supersonic. The new results are as follows: (i) Using an analogy between the "granular-gas" state and the Knudsen regime $[11,14,15]$ of a ideal gas, it is demonstrated that classical hydrodynamics cannot be used. (ii) An analogy with the thermodynamics of a single bead is then drawn. (iii) It is proposed that the $p \sim(A \omega)^{3 / 2}$ scaling observed experimentally is linked to the crossover from a regime of single collision during the sampling time, which imposes $\mathrm{p} \sim \mathrm{A} \omega$, to a multiple-collision regime imposing $\mathrm{p} \sim \mathrm{A}^{2} \omega^{2}$.

In a second part, we present new results on sedimentation under vertical vibration which proves that vibration can produce linear or planar structures perpendicularly to the vibration. Vibration could then be used to structure an heterogeneous fluid, within some conditions.

\section{SOME BASIC ANALOGIES FOR GRANULAR GAS EXPERIMENTS}

The Mini- Texus 5 space-probe was launched on 11/2/1998 from Esrange (Northern Sweden) with 3 cubic containers on board, $\mathrm{L}^{3}=1 \mathrm{~cm}^{3}$ in inner volume, with 2 transparent sapphire walls. Each cell is filled, respectively, with $0.281,0.562$ and $0.8915 \mathrm{~g}$ of $\mathrm{d}=0.35 \pm 0.05 \mathrm{~mm}$ in diameter bronze spheres. Thus, the total number of particles in each cell is about $\mathrm{N}_{0} \approx 1420,2840$ and 4510 , corresponding to roughly $1-, 2$ and 3- particle layers at rest with a mean free path $l_{c}=L, L / 2$ and $L / 3$, since $\pi d^{2} l_{c} N_{0} L^{3} \approx 1$. An electric motor, with eccentric transformer from rotational to translation motion, drives the vessels sinusoidaly in the $z$ direction at frequency $\mathrm{f}=\omega /(2 \pi)$ and maximum-displacement amplitude $A$ in the ranges 1 to $60 \mathrm{~Hz}$ and 0.1 to $2.5 \mathrm{~mm}$ respectively $(\mathrm{z}=\mathrm{A} \cos [\omega \mathrm{t}]), c f$. Table 1 for the precise set of values. A video camera with a resolution $742 \times 582$ pixels was used to observe the particle dynamics; the cell motion was controlled via a 3-axis accelerometer and the granular pressure was measured in the $z$ direction with 3 gauges $(1.53 \mathrm{~cm}$ in diameter). See refs. [6,7] for more information about experimental set-up. Data were 
obtained with a $\tau_{\mathrm{s}}=0.5 \mathrm{~ms}$ sampling time during the 200 seconds of low gravity environment and were transferred to Earth in real time.

Table 1. Typical number $n_{c}$ of collisions per acquisition time $\left(\tau_{s}=0.5 \mathrm{~ms}\right)$, typical mean expected pressure $\mathrm{p}_{\mathrm{c}}$, and typical losses (collisional vs. viscous) as a function of the experiment number \#. (See Eq. 4 for the definition of $n_{c} \& p_{c}$ ).

\begin{tabular}{|c|c|c|c|c|c|c|c|c|c|}
\hline $\begin{array}{c}\exp \# \\
A(\mathrm{~mm}) \\
\mathbf{F}(\mathbf{H z})\end{array}$ & $\begin{array}{c}1 \\
0.1 \mathrm{~mm} \\
3 \mathrm{~Hz}\end{array}$ & $\begin{array}{c}2 \\
2.5 \mathrm{~mm} \\
1 \mathrm{~Hz}\end{array}$ & $\begin{array}{c}3 \\
2.5 \mathrm{~mm} \\
3 \mathrm{~Hz}\end{array}$ & $\begin{array}{c}4 \\
0.3 \mathrm{~mm} \\
30 \mathrm{~Hz}\end{array}$ & $\begin{array}{c}5 \\
0.1 \mathrm{~mm} \\
60 \mathrm{~Hz}\end{array}$ & $\begin{array}{c}6 \\
0.3 \mathrm{~mm} \\
60 \mathrm{~Hz}\end{array}$ & $\begin{array}{c}7 \\
1 \mathrm{~mm} \\
60 \mathrm{~Hz}\end{array}$ & $\begin{array}{c}8 \\
2.5 \mathrm{~mm} \\
30 \mathrm{~Hz}\end{array}$ & $\begin{array}{c}9 \\
2.5 \mathrm{~mm} \\
60 \mathrm{~Hz}\end{array}$ \\
\hline $\mathbf{n}_{\mathrm{c}}$ & 0.04 & 0.35 & 1 & 1.26 & 0.84 & 2.5 & 8.4 & 10.5 & 21 \\
\hline $\mathbf{p}_{c}$ & 0.01 & 0.08 & 0.24 & 0.29 & 0.19 & 0.58 & 1.9 & 2.4 & 4.8 \\
\hline $\mathbf{p}=\mathbf{n}_{\mathbf{c}} \mathbf{p}_{\mathrm{t}}$ & 0.0004 & 0.03 & 0.24 & 0.37 & 0.16 & 1.5 & 16 & 25 & 101 \\
\hline losses & Large viscous & Large viscous & viscous & viscous & viscous & viscous & viscous & viscous & collisional \\
\hline
\end{tabular}
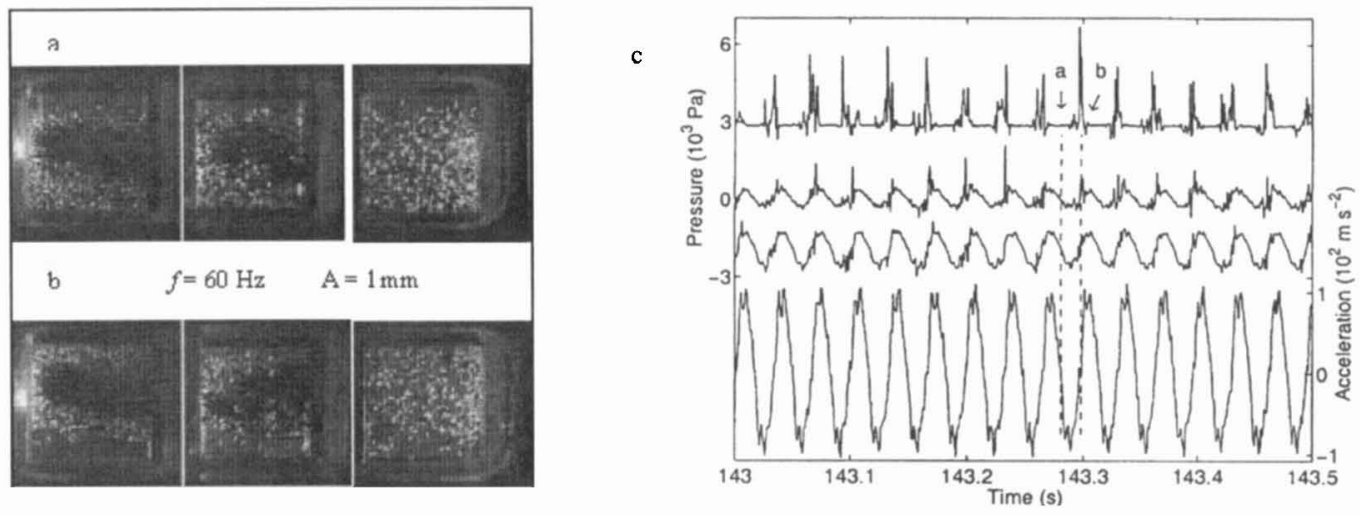

Figure 1. Experimental results for $A=1 \mathrm{~mm}, \mathrm{f}=60 \mathrm{~Hz}$ : Fig. 1.a \& 1.b: Transition between gas behaviour (right) to cluster behaviour (left \& middle). The right cell is the most dilute, the left one the densest. The pressure sensor is on "top" of the cells. (a) "upward" velocity. (b) "downward" velocity. Fig. 1c: From top to bottom, time dependence of the pressure in the mostdilute cell, medium-dense cell, densest cell and z-acceleration. Pressure curves are shifted vertically for clarity. Spikes of top curve demonstrate that the mean velocity of the granular particles of the dilute regime is smaller than sensor speed Aw.

Two typical observations are reported in Fig. 1.a \& 1.b when the cells are at maximum displacement and in opposite phase. Similar results have been obtained for the other sets of parameters. One observes the formation of a dense cluster in the two densest cells but not in the third one. So, cluster formation occurs as soon as the mean free path $\mathrm{l}_{\mathrm{c}}$ between two grain-grain collisions is smaller than the cell size $\mathrm{L}$. In the three cells one observes also a loose granular medium which fills the cell, except in a small region near the wall which moves away from the gas. This depletion zone is the proof that cell moves faster than the grains and that the excitation is "supersonic"; from the duration of the depletion, one can estimate the mean speed $\underline{\mathrm{v}}$ of the grains to be $\underline{\mathrm{v}}=\mathrm{A} \omega / 4$ about. This result is confirmed by the pressure signal of the most dilute sample (Fig. 1.c), which exhibits periodic bursts of pressure when the gauge meets the "granular-gas", and no signal when the gauge is in the depletion zone [7].

Some dilute gas is also observed all around the cluster in the denser cells. The gas state of the dilute cell can be called the "granular-gas" regime; however, it is worth noting that this gas is in a very peculiar state, since the mean free path $k_{c}$ of the particles is larger than or equal to the cell size $\mathrm{L}$ - the so-called Knudsen regime $\left(l_{c}>L\right)[7,11,15,16]$.

One can compute the pressure distribution [6,7] generated in this dilute cell for each set of parameters $(A, \omega)$. A rescaling procedure [6] demonstrates that $p$ scales rather as $(A \omega)^{3 / 2}$ in the dilute cell and not as $\mathrm{p} \sim(\mathrm{A} \omega)^{2}$. In [6], this result was interpreted as generated by a speed dependence of the restitution coefficient $\varepsilon=r^{2}$, where $\varepsilon$ is defined as $\varepsilon=\left(\Delta v^{\prime} / \Delta v\right)^{2}$, i.e. it is the square of the ratio of the relative speed after $\left(\Delta v^{\prime}\right)$ and before $(\Delta v)$ the collision. We propose here other interpretations. 
Can we understand these results within simple models? Two simple models can be invoked. If one neglects the grain-grain collision, the system simplifies into the problem of a single particle in a vibrated container, which will be our first model [10]. The second model is the Knudsen gas.

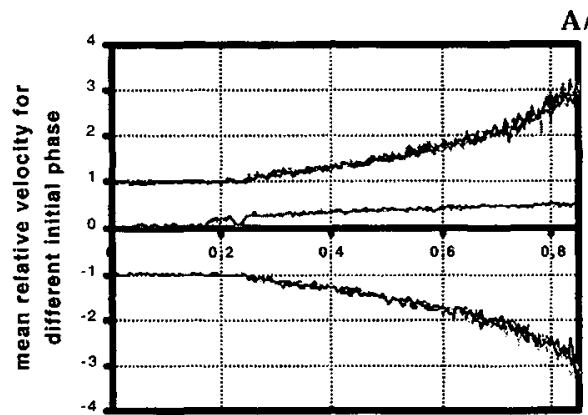

square root of restltution coefficient

$\mathrm{A} / \mathrm{L}=\mathbf{0 . 0 0 1}$

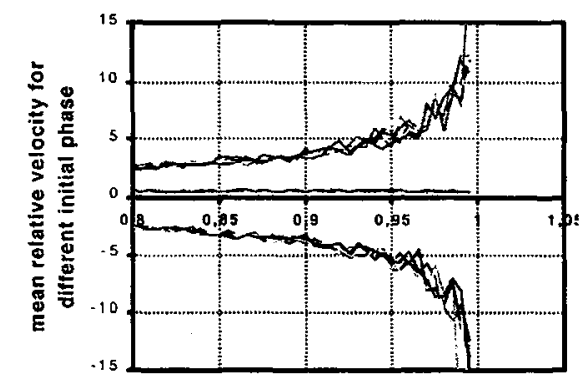

square root of restitution coeftlcient

Figure 2. Mean relative speed $\underline{v} /(A \omega)$ and width $\delta v / \underline{y}$ of a single grain in a $1 \mathrm{~d}$ container as a function of $\varepsilon^{1 / 2}=r=-\Delta v^{\prime} / \Delta v$, for different initial conditions. Left: $r<0.8$, Right $r>0.8$. One remarks that $\underline{y} \geq A \omega$ and that $\delta v / \underline{y} \approx 0.3$. Resonance is visible for r $>0.95$ (right Fig.), (Figs. from [10]).

\subsection{Analogy with a single particle in a vibrating $1 \mathrm{~d}$ cell}

As the "granular-gas" is very dilute the simplest modeling is to consider that grain-grain collisions can be neglected. In this case the problem is analogous to the one of the thermodynamics of a single bead in a vibrating box, which has been studied in $1 \mathrm{~d}$ in [10]. In the limit of small amplitude, i.e. $\mathrm{A} / \mathrm{L} \ll 1$, and of small enough $\mathrm{r}$, i.e. $\mathrm{r}<0.95$, the $1 \mathrm{~d}$ problem can be solved using the random phase approximation [10] which states no phase memory between two collisions. One finds [10] a mean velocity $\underline{v}$ :

$$
\underline{\mathrm{v}} \approx \mathrm{A} \omega(1+\mathrm{r})^{1 / 2} /[2(1-\mathrm{r})]^{1 / 2}
$$

which is in good agreement with the direct numerical simulations reported in Fig. 2 from ref. [10]. One finds also the typical width distribution $\delta \mathrm{v} / \underline{\mathrm{y}}$ is 0.3 about and the bead dynamics is ergodic and does not depend on the initial conditions in this range of parameters. However, when $A / L$ is larger than 0.005 and $r>0.95$, the dynamics is disturbed:

- When $r>0.95$, some resonant effect is found at large $\mathrm{A} / \mathrm{L}$, which makes the dynamics depending on the initial condition: the distribution of possible speeds becomes peaked around a discrete set of fixed values which depend on $\mathrm{A} / \mathrm{L}$ and $\mathrm{r}$. The relative width of each peak is small proving the resonance. This forces the dynamics to become non ergodic, especially for some specific values of $(A / L, r)$.

- As there are two lengthscales $A \& L$, the ratio $A / L$ can be important. As can be seen on Fig. 3 , the simulations show that the mean relative speed $\mathrm{v} /(\mathrm{A} \omega)$ depends on $\mathrm{A} / \mathrm{L}$ when $\mathrm{A} / \mathrm{L}$ becomes larger than 0.005 . According to this Fig. $3, \underline{v} /(\mathrm{A} \omega)$ decreases when $\mathrm{A} / \mathrm{L}$ increases in the range $\mathrm{A} / \mathrm{L}=[0.01,0.2]$ which is the experimental range of the MiniTexus-5 experiments. So, this effect may contribute to explain the strange experimental scaling $p-A^{3 / 2} \omega \omega^{3 / 2}$ instead of the expected one $p-A^{2} \omega^{2}$ because it implies a decrease of the $A$ exponent. However it cannot explain the anomalous $p-\omega^{3 / 2}$ dependence since the experiment has no specific time scale.

-A point which remains not explained within this model is the supersonic nature of the excitation. It is clear from Fig. 1 that the cell moves faster than the beads in the experiment. This is not what it has been found from the simulations of a single-particle in a $1 \mathrm{~d}$ box [10].

In order to explain this fact, i.e. $\underline{\mathrm{v}}<\mathrm{A} \omega$, one can invoke the effect of grain-grain collision. Indeed, if the grain-grain collisions have no real effect on the mean dynamics when the system is strictly 1d [12], it is not the case as soon as the real system is $2 \mathrm{~d}$ or $3 \mathrm{~d}$ : the final speeds after collision depend not only on 
the initial speeds but also on a "hidden" parameter which is called the impact parameter. It results from this impact parameter that the speed of a particle is redistributed (more or less) randomly in the other $(\mathrm{x}, \mathrm{y})$ directions after each grain-grain collision, which reduces efficiently the bead speed in the $z$ direction. This process may then explain the value of the observed speed, i.e. $\underline{\mathrm{V}}<\mathrm{A} \omega$, as soon as the mean free path $\mathrm{l}_{c}$ of the particle becomes of the order of the cell size $L$.

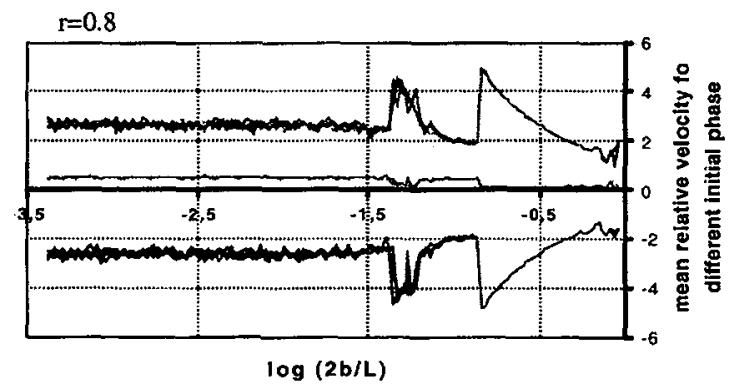

Figure 3. Simulation of the dynamics of a single bead in a 1d box: Evolution of the mean relative speed $\underline{v} /(\mathrm{A} \omega)$ as a function of $\ln (2 \mathrm{~A} / \mathrm{L})$ for $r=\varepsilon^{1 / 2}=0.8$. One observes a finite size effect at large $A / L$. Other simulations can be found in [10].

\subsection{Viscous effect}

Another possible mechanism of speed reduction is viscous damping due to air, which applies a drag force $F_{v}=-6 \pi \eta R v$ on each grain where $\eta=1.810^{-3} \mathrm{~g} \cdot \mathrm{mm}^{-1} \cdot \mathrm{s}^{-1}$ is the air viscosity, $R=0.15 \mathrm{~mm}$ the grain radius, $m \approx 0.2 \mathrm{mg}$ the grain mass. Solving $F_{v}=m \mathrm{dv} / \mathrm{dt}$ leads to the dynamics:

$$
v=v_{0} \exp [-\alpha \mathrm{t}], \quad \text { with } \alpha=6 \pi \eta R / \mathrm{m}=0.27 \mathrm{~s}^{-1}
$$

Three different regimes can be considered depending on the damping time $\alpha^{-1}$ compared to the three other parameters of the experiments, i.e. the restitution coefficient $r=-\Delta v^{\prime} / \Delta v$, the period $1 / f$ of vibration and the time $\mathrm{T}_{\mathrm{r}}=\mathrm{L} /(\mathrm{Af})$ the particle needs to fly over the cell:

- if $f<\alpha$, the beads follow the periodic motion of the box due to the coupling via the air; this occurs when $\mathrm{f}<1 / 4 \mathrm{~Hz}$. So, it cannot be observed during the MiniTexus- 5 experiments.

- $\alpha L /(A f)<-\ln (r)$ : viscous losses are negligible compared to grain-wall collision losses. Assuming $\mathrm{r}=0.98$ leads to conclude that this case is obtained when $0.1>\mathrm{L} /(\mathrm{Af})$, which is never the case during MiniTexus5 flight except for experiment \#9.

- when $f=\alpha(2 \pi)>\alpha$ and $\alpha L /(A f)>-\ln \varepsilon$, the viscous damping generates the main losses. According to Fig. 1 , the typical speed of the grain is $\mathrm{v} \approx \mathrm{A} \omega / 4$; so the typical flying time between two collisions with the walls is $T_{c}=4 L /(A \omega)$, leading to a velocity reduction which is at first order $v / v_{0} \approx \exp \left[-\alpha T_{c}\right] \approx \exp \left[-4 \alpha T_{c}\right.$ $L /(A \omega)]$. So the viscous damping can be very efficient or not depending whether $4 \alpha L /(A \omega)$ is larger than 1 or not. In the first case, air damps strongly the dynamics, whereas it does not in the second case. The first case corresponds to MiniTexus5-experiment \#1 \& 2, whereas the other case to experiment \# 38. These results are summarised in Table 1.

Indeed, from an experimental point of view, we observe, $c f$. Fig. 1, that the larger the amplitude and the frequency the smaller the viscous damping and the better the observation of the depletion zone. So, the above analysis demonstrates unambiguously that the viscous damping of the particle motion due to the interaction with air is not responsible for the supersonic nature of the wall motion.

\subsection{Analogy with a gas in the Knudsen regime}

The granular-gas which has been studied during the MiniTexus-5 experiments corresponds to the socalled Knudsen regime [11]. It implies that the mean free path $\mathfrak{k}$ between two grain-grain collisions is larger than the cell size L. This regime is quite specific since one can define local averages such as 
density $\rho$, pressure $p$, temperature $T$, which have some meaning according to the ergodic principle. However, these variables cannot be used to predict the sample evolution using classical hydrodynamics equations because this formalism describes the evolution of each elementary volume as depending only on the adjacent elementary volumes. On the contrary, in the Knudsen regime, the interactions are no more local but cover the whole sample. This is why it is difficult to speak in term of wave propagation and of hydrodynamics.

However, it is worth noting that the sound propagation has been studied in gases under very low pressure $[15,16]$ even when $L<t[15,16]$ using two parallel plates at temperature $T$. In this microscopic regime, the statistics of the response to a vibration excitation of a plate can be calculated and compared to experiments. It is found that most of the atoms transfer directly their momentum to the walls, and that only some of them collide together.

- Representative elementary volume REV: At this stage it is worth discussing what is the size of the REV. As mentioned above, the REV $\left(\varpi_{0}\right)$ above which classical hydrodynamics equations hold is defined by $\varpi_{0} \approx l_{c}^{3}$. Since $l_{c} \approx 1 /\left(n \pi d^{2}\right)$, where $n$ is the particle density, the REV $\varpi_{0}$ scales as $\varpi_{0} \approx 1 /\left(\pi^{3} d^{6} n^{3}\right)$; hence it contains in mean $N_{c} \approx 1 /\left(\pi^{3} d^{6} n^{2}\right)$ molecules. So the number of particles in the REV increases strongly when the system is rarefied as in a gas. On the contrary, one gets $N_{c} \approx 1$ when the system is dense as in the case of a liquid or of a solid. This effect seems to have been completely ignored in the granular-matter area, where it is often asserted that small REV is the requirement to get classical physics. On the other hand, the above description shows that the local average of the thermodynamics parameters are preserved at scales smaller than $l_{c}$ according to the ergodic principle; thus the $\mathrm{pv}=\mathrm{nRT}$ law is valid at small scale.

So, we can predict what the pressure signal would be in the case of a Knudsen gas with supersonic excitation. Let us first consider a non-moving pressure gauge of surface $\Sigma$ during a time $\delta$ t; the gauge receives $\mathrm{N}$ collisions during $\delta \mathrm{t}$, which generates the pressure $\mathrm{p}$, such as $\mathrm{p} \Sigma \delta \mathrm{t}=\mathrm{N}(2 \mathrm{~m} \underline{\mathrm{v}})=2 \mathrm{~m} \underline{\mathrm{v}} \mathrm{n} \underline{\mathrm{v}} \Sigma \delta \mathrm{t}$, since $N=n \underline{v} \Sigma \delta$ t is the average number of particles colliding with the gauge. So one gets the average pressure $\mathrm{p}_{0}$ :

$$
\mathrm{p}_{0} \approx 2 \mathrm{~m} \underline{\mathrm{v}}^{2} \mathrm{n}
$$

According to the central limit theorem, when all the particles move independently from one another, the statistical fluctuations $\Sigma \Delta \mathrm{p}$ during $\delta \mathrm{t}$ of the force $\mathrm{F} \delta \mathrm{t}=\Sigma \mathrm{p} \delta \mathrm{t}$ scales as $\mathrm{N}^{1 / 2} \delta(2 \mathrm{mv})$, where $\delta(\mathrm{mv})$ is the typical fluctuation of particle momentum. As, for a gas $\delta(\mathrm{mv}) \approx \mathrm{m} \underline{\mathrm{v}}$, one gets the typical mean pressure $\langle\mathrm{p}\rangle$ :

$$
\Delta(\mathrm{p} \Sigma \delta \mathrm{t}) \approx \mathrm{m} \underline{\mathrm{v}}^{3 / 2} \mathrm{n}^{1 / 2}(\Sigma \delta \mathrm{t})^{1 / 2} \quad \text { when } \mathrm{N} \text { is large }
$$

As far as the duration $\delta t$ and the surface $\Sigma$ are large enough, the number of collisions per $\delta \mathrm{t}$ is much larger than 1. In this case, one expects that the density distribution of the signal is Gaussian, i.e. $\rho(p)-\exp \left\{-\left(p-p_{o}\right)^{2} /(\Delta p)^{2}\right\}$; it is distributed around its mean value $p_{o}$ which is given by Eq. (3.a), and its typical width $\Delta p$ is given by Eq. (3.b). This is a consequence of the central limit theorem and of the fact that the motions of the molecules are not correlated.

The Maxwellian distribution, predicted by the kinetics theory of gas, i.e. $\rho\left(v_{z}\right) \sim \exp \left[-\mathrm{mv}_{\mathrm{z}}{ }^{2} /(2 \mathrm{kT})\right]$, is only observable when the number of collisions during $\delta t$ is smaller than 1 . In this case the pressure is proportional to $\mathrm{mv}_{\mathrm{z}}$ and the pressure distribution shall then scale as $\rho(\mathrm{p}) \sim \exp \left[-\mathrm{p}^{2} /(2 \mathrm{mkT})\right]$. Hence it is still a Gaussian, but it is centred on $\mathbf{0}$. So, in the case of ideal gases of atoms or molecules, $\rho(p)$ is always Gaussian-shaped when the pressure is measured with an immobile sensor; it is then a general feature, which is independent of $l_{c}$ and of the density of the gas; it applies in the Knudsen regime as well as in a denser case. Only the mean $p_{o}$ and the width $\Delta p$ of the Gaussian do depend on the size of the measuring surface and on the integration time. Moreover $\mathrm{p}_{\mathrm{o}}$ and $\Delta \mathrm{p}$ do not depend on $\mathrm{l}_{\mathrm{c}}$.

\section{4 pressure distribution in the case of a periodic supersonic excitation by the gauge}

Starting with a subsonic gauge motion, one obtains that the transfer of momentum is proportional to the relative speed of the particle in the gauge frame. So, one expects a sinusoid response if the gauge moves 
sinusoidaly; but the complete response of the gas depends also on the cell size compared to the wavelength of the sound [17].

Anyway, when the sinusoidal motion of the gauge is supersonic, no particle can hit the gauge during some part of the period; the larger pressures are obtained when the gauge moves in the direction of the gas. As the amplitude of variation of the gauge speed is much larger than the fluctuation of particle speed, the distribution reflects the distribution of speed of the gauge: a large pressure signal corresponds to fast gauge motion towards the gas whereas small pressure signal refers to smaller gauge speed and zero pressure signal to gauge moving backward from the gas. However, the pressure signal itself can be proportional to $m v_{\text {cell }}$ or to $\mathrm{mv}_{\text {cell }}{ }^{2}$ depending on the number $\mathrm{n}_{\mathrm{c}}$ of collision occurring during the sampling time $\tau_{\mathrm{s}}$ according to:

$$
\begin{aligned}
& \text { for } n_{c}<1 \quad \Rightarrow p_{c} \approx m v_{\text {cell }}\left(\text { with probability } n_{c}\right) \quad \text { and } p_{c}=0 \quad \text { (with probability } 1-n_{c} \text { ) } \\
& \text { for } n_{c}>1 \quad p=n_{c} p_{c} \approx m v_{c e l l}{ }^{2}
\end{aligned}
$$

This is just what one shall expect also for MiniTexus 5 experiments. According to Fig. 1 at each period the granular cloud expands over the amplitude $A$ about on both sides, leading to a number $N_{c}$ of collisions per period given approximately by $N_{c} \approx A N_{0} /(2 L)$, where $N_{0}$ is the total number of particles in the cell, i.e. $\mathrm{N}_{0}=1420$. According to Fig. 1 also, these collisions occur during about $14^{\text {th }}$ of the period. It means that the number $n_{c}$ of collisions for each sampling time $\tau_{s}=0.5 \mathrm{~ms}$ is $n_{c} \approx 2 \mathrm{AN}_{\mathrm{o}} f \tau_{\mathrm{s}} / L=1.4 \mathrm{Af} / \mathrm{L}$, (where 1.4 is in s). Table 1 reports the expected values of $n_{c}, p_{c m}$ and of the typical maximum pressure $\mathrm{p}=\mathrm{n}_{\mathrm{c}} \delta \mathrm{p}_{\mathrm{cm}}$ for the different experimental cases.

So, one expects from Table 1, that experiments \# 1-5 are concerned with less than 1 collision per sampling time whereas experiments \# 6-9 are concerned with more than 1 collision per sampling time. One expects that the recorded pressure scales as $p \sim m(1+r)\left(v+v_{\text {cell }}\right)$ in the first case and as $\mathrm{p} \sim \mathrm{m}(1+r)\left(v+v_{\text {cell }}\right)^{2}$ in average in the second case. One therefore understands the $\mathrm{p} \sim(\mathrm{A} \omega)^{3 / 2}$ experimental fit as resulting from a compromise between the two previous scalings $A \omega \&(A \omega)^{2}$.

One should remark also that neither the real mean speed $\underline{v}$ of the particle nor its dispersion have some importance since the pressure is dominated by the speed of the impact which is mainly the cell speed in the case of "supersonic excitation".

\subsection{Effect of g-jitter}

In the case of the MiniTexus 5 experiments, one can estimate the fluctuations of $\mathrm{g}$ to be less than $10^{-5} \mathrm{~g}=$ $10^{-4} \mathrm{~m} / \mathrm{s}^{2}$ in the range $100 \mathrm{~Hz}-1 \mathrm{~Hz}$. So, the high frequency g-jitter, i.e. $\mathrm{f}>1 \mathrm{~Hz}$ is quite negligible compared to the mechanical noise of the vibrating device itself. The low-frequency component of the gjitter shall be even much smaller than $10^{-4} \mathrm{~m} / \mathrm{s}^{2}$ since the rocket trajectory is compensated. However, this low frequency noise shall force the cluster to perform a random walk whose time step is $\tau_{\mathrm{w}}=1 / \alpha \approx 4 \mathrm{~s}$ about and its path length is probably smaller than $1 \mathrm{~mm}$.

Taking into account the typical flying time between the walls $\tau_{f} \approx L(A f)$, the random walk process becomes non negligible only when $\tau_{\mathrm{f}}>\tau_{\mathrm{w}}$, which imposes $\mathrm{Af} / \mathrm{L}<0.2 \mathrm{~s}^{-1}$. According to Table 1 , this is the case for experiment \# 1 only.

\subsection{Discussion and conclusion}

Additional experiments were performed in the Airbus A300 $0 \mathrm{~g}$ of CNES. They were concerned with the "granular-gas" case in very dilute condition. Preliminary results, which use an immobile force sensor confirm the results, i.e. supersonic excitation. In what concern the study of the cluster dynamics, it is more sensitive to small perturbation: indeed, Fig. 1 indicates that the cluster moves much slower than the particles of the "granular-gas". So its travel time $T_{t}$ from one side of the cell to the other one is quite large. It results from this that its dynamics is much more disturbed by the viscous damping and depends strongly on the g-jitter. Due to this limitation, the study of the typical evolution of the cluster requires long period of micro-gravity environment, and small $\mathrm{g}$-jitter, conditions which cannot be obtained in the Airbus $\mathrm{A} 300 \mathrm{Og}$. Its study requires rocket flights and/or experiments in the space station. 


\section{SEDIMENTATION UNDER VIBRATION}

In general, one performs experiments in micro-gravity to get rid of $\mathrm{g}$ induced perturbation. However, quite often the g-sensitivity of the experiment comes from a heterogeneous distribution of density. The system is thus sensitive also to g-fluctuation and hence to vibration. This is why it becomes quite important to study the effect of vibration in most micro-g experiments.

Fig. 4 shows different patterns obtained when small particles sedimentate from a hole in either a cylindrical column or in a flat hourglass. In this last case, the particles used were less dense than the surrounding liquid so that they move spontaneously upward instead of falling down. When no vibration is applied, i.e. Fig. 4.a \& 4.c, the particles move vertically remaining in the central part of the system. But as soon as vertical vibration of rather large amplitude and frequency, i.e. $\mathrm{A}>2 \mathrm{~mm}, \mathrm{f}>10 \mathrm{~Hz}$, is applied one observes a horizontal layering (Fig. 4.b, 4.d, 4.e \& 4.f), which can be more or less complex. The typical thickness of a layer is one-particle diameter, but its length can spread over the cell diameter. One remarks also that visualisation of the phenomenon is easier in $2 \mathrm{~d}$ than in $3 \mathrm{~d}$; this is likely due to the convex shape of the layer which is more pronounced in $3 \mathrm{~d}$ than in $2 \mathrm{~d}$. This is why also the phenomenon is quite difficult to observe when starting from a $3 d$ homogeneous sedimenting medium. The qualitative explanation of this pattern can be found in [18]. Here it was assumed that the liquid was viscous enough to get a large viscous boundary layer $\delta=(v / \omega)^{1 / 2}, v$ being the kinematic viscosity; so if the sedimenting medium is dense enough, and the bead small enough, the mean distance between the beads is smaller than $\delta$ and the sediment flows as a homogeneous fluid inside the liquid. The action of vibration is to induce a periodic flow $u(t)=u_{0} \sin (\omega t)$ and a periodic Bernoulli pressure $\mathrm{p}=-\mathrm{u}_{0}{ }^{2} \sin ^{2}(\omega \mathrm{t})$, which exhibits a non zero mean. This periodic forcing is then able to generate an instability of the Kelvin-Helmholtz (KV) type similar to the one reported in [19]. When fully developped the $\mathrm{KV}$ instability generates horizontally stratified layering perpendicularly to the vibration.

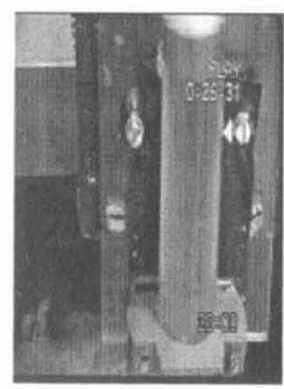

(a)

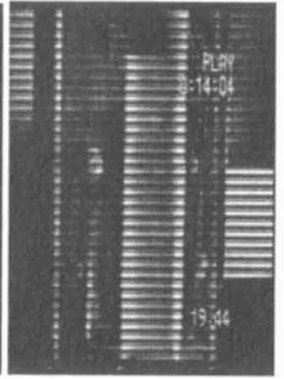

(b)

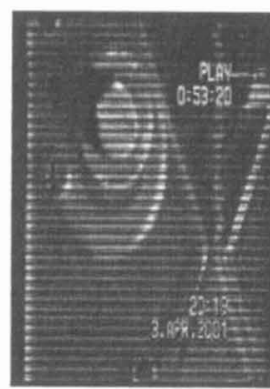

(c)

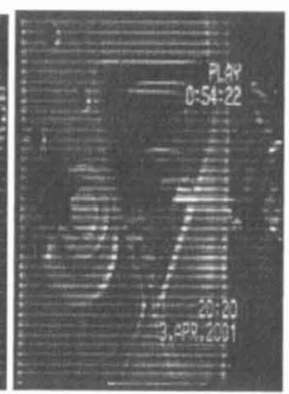

(d)

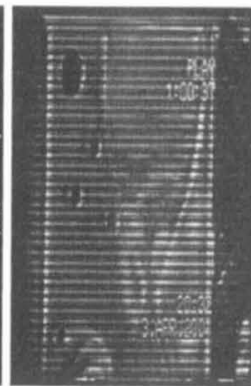

(e)

Figure 4: Normal sedimentation pattern obtained from a hole (a) in $3 d \&$ (c) in $2 d v s$. horizontal-layering patterns obtained during sedimentation under vertical vibration (b), (d) \& (e) with the same devices as in (a), (c). The patterning is due to an instability of the Kelvin-Helmholtz kind $[18,19]$, which persists also near a critical phase transition [20].

\section{CONCLUSION}

From the different examples reported here, it is obvious that granular matter is a rich topics which deserves to be studied in weightlessness condition. It is also clear that vibration may induce important experimental effects in micro-gravity. Such effects deserve to be studied at least to remain under control, and to be used for handling fluids in space. These effects are quite general, they can also be observed near a critical point [20]. Another point, which has to be noticed, is the fact that non linear vibration, such as rotation vibrations, can generate new forces and attract the denser parts of the fluid at the centre of rotation [21]. This is another story, for future investigation. It is obvious that this science and this technique will benefit from the building of a centrifuge in space since it is the first step to allow studying the combination of two inertial effects with strong non linear coupling. 


\section{Acknowledgements}

The authors wants to thank C. Chabot-Lecoutre \& R. Wunenburger for their help during the whole launching campaign and for discussions. Collaboration with E. Falcon and S. Fauve has been quite appreciated, in particular for the statistical treatment of the pressure signals and for clever ideas. Collaboration and discussions with V.G. Kozlov, T. Lubimova, D. Lubimov and B. Roux have been appreciated.

CNES is thanked for funding and ESA for providing the experiment and the flight opportunity; Mini-Texus 5 sounding rocket is a program of E.S.A.; MiniTexus 5 was launched from Esrange on February 1998. The bronze spheres have been provided by Makin Metal Powders Ltd. The experiment module has been constructed by D.A.S.A. (Germany), Ferrari (Italy), and Techno System (Italy). We gratefully acknowledge the Texus team for its kind technical assistance.

\section{References}

1. Worms J.C., de Maximy H., réalisation Ch. Bargues, "Une loi réputée simple: pV=nRT"; (Planet 6 \& CNES ed., Planet 6 c/o J.C. Worms, 12 rue de l'Espérance, 67400 Illkirch, France, 1993)

2. Pöschel T. \& LudingS. eds., Granular gases, (Lecture Notes in physics series, Springer, Berlin, 2001), and refs. there in.

3. Du Y., Li H. \& Kadanoff L.P., Phys. Rev. Lett. 74,1268, (1995).

4. Goldhirsch I., in Granular gases, (Lecture Notes in physics series, Springer, Berlin, 2001), pp. 79-99, and refs. there in.

5. Goldshtein A., Alexeev A., \& Shapiro M., in Granular gases, (Lecture Notes in physics series, Springer, Berlin, 2001) , pp. 266-277

6. Falcon E., Wunenburger R., Évesque P., Fauve S., Chabot C., Garrabos Y. and Beysens D., Phys. Rev. Lett. 83, 440-443, (1999)

7. Évesque P., Falcon E., Wunenburger R., Fauve S., Lecoutre-Chabot C., Garrabos Y. and Beysens D., in Proc. of the "First international Symposium on Microgravity Research \& Applications in Physical Science and Biotechnology", 10-15 Sept 2000, Sorrento, Italy, (B. Schürmann ESA ed. , 2001), pp.829-834

8. Goldreich P. and Tremaine S., Ann. Rev. Astron. Astrophys. 20, 249, (1982).

9. Kudrolli A., Wolpert M. and Gollub J.P., Phys. Rev. Lett. 78, 1383, (1997).

10. Évesque P., poudres \& grains 12, 17-42, (2001), http://prunier.mss.ecp.fr/poudres\&grains/poudresindex.htm

11. Hirschfelder J.O., Curtis C.F. \& Bird R.B., Molecular theory of gases and liquids, (John Wiley \& sons eds, New York, 1967), pp. 694-704

12. Évesque P., poudres \& grains 12 (3), 50-59, (2001), http://prunier.mss.ecp.fr/poudres\&grains/poudresindex.htm

13. Falcon E., Laroche C., Fauve S. and Coste C., Eur. Phys.J. B 3, 45 (1998).

14. Landau L. \& Lifschitz E., Mécanique des Fluides (cours de Physique tome 6, Mir, 1989)

15. Greenspan M., in Physical Acoustics, tome 2-A, (W.P. Mason ed., Academic press,1965), 1-45

16. Cook R.K., Greenspan M. \& Thompson M.C. Jr., J. Acoust. Soc. Am. 25,192, (1953)

17. Évesque P., poudres \& grains 12 (4) 60-82, (2001), http://prunier.mss.ecp.fr/poudres\&grains/poudresindex.htm

18. Évesque P., Pour la Science 239, 94-96, (1997)

19. Ivanova A., Kozlov V. \& Évesque P., Europhys Lett. 35(3), 159-164 , (1996)

20. Wunenburger R., Évesque P., Chabot C., Garrabos Y., Fauve S. \& Beysens D., Phys Rev. E 52 , 5440-5445, (1999)

21. Ivanova A.A., Kozlov V.G., Évesque P., Fluid Dynamics 33, 488-496, (1998) 